\title{
Bayesian generalized linear model for identifying predictors of child nutritional status in Ethiopia
}

\begin{abstract}
Background: In Ethiopia, child malnutrition is one of the most serious public health problem and the highest in the world. The overall prevalence of underweight among children under five years old in Ethiopia was 25\% and $7 \%$ are severely and moderate underweight in 2014 respectively. Malnutrition in sub-Saharan Africa contributes to high rates of childhood morbidity and mortality. However, little information on the nutritional status of children is available from informal settlements. The primary aim of this study was then to determine the determinants of children malnutrition in Ethiopia using Bayesian generalized linear model. The overall prevalence of underweight among children in Ethiopia was $36.4 \%$.
\end{abstract}

Methods: Data was obtained from 2011 Ethiopian Demographic and Health Survey (EDHS). Bayesian Generalized regression model was used to identify the effects of selected socioeconomic, demographic, health and environmental covariates. Bayesian approach with Markov Chain Monte Carlo (MCMC) technique was used.

Results: The analysis result revealed that out of the 11, 654 number cases examined in this study $32.8 \%$ of male children were underweight and $33.6 \%$ of female children were underweight. It was found that the covariates succeeding birth interval, sex of child, child by choice not by chance, vaccination and cough were the most important determinants of children nutritional status in Ethiopia.

Conclusion: Inference is the fully Bayesian and classical generalized linear model based on recent Markov chain Monte Carlo techniques.Some of the socioeconomic, demographic and environmental determinants included in the study were found to be statistically significant. The result was suggested that for reducing childhood malnutrition, due emphasis should be given in improving the knowledge and practice of parents on appropriate young child feeding practice and frequent growth monitoring together with appropriate and timely interventions.

Keywords: nutrition, weight-for-age, malnutrition, anthrometric measurement, mcmc, bayesian generalized linear model
Volume 8 Issue 2 - 2019

\author{
Reta Habtamu Bacha, Megersa Tadesse \\ Department of Statistics, Jimma University, Ethiopia \\ Correspondence: Reta Habtamu Bacha, Department of \\ Statistics, Jimma University, Jimma, Ethiopia, \\ Email habtamuretab@yahoo.com
}

Received: December 29, 2018 | Published: April 30, 2019

\section{Background}

Malnutrition is the underlying cause of more than 2.6 million child deaths each year. Globally significant numbers of people are affected by malnutrition(undernutrition). The Food and Agriculture Organization(FAO) estimated that undernutrition affects approximately 789 million people in 2014-16. ${ }^{1,2}$ The prevalence of undernourshment and underweight among children under age five were primarily used internationally to measure and compare the burden of undernutrition. ${ }^{2}$

The global trend in undernourishment has shown a substantial improvement. $^{3}$ The total number of people undernourished in developing countries significantly decreased from 990 million people in 1990-92 to 789 million people in 2014-16. However, a closer look at the change in the proportion of undernourishment over the years tells us that this has not changed in developing countries. There is a wide variation in prevalence of undernourshment among regions. Two regions, southern Asia and Sub-Saharan Africa account for approximately $35.4 \%$ and $27.7 \%$ of the shares of global undernourishment, respectively. ${ }^{2}$ Next to southern Asia, Sub-Saharan African countries, especially eastern African countries, have the highest burden of undernutrition than any region in the world. The continent of Africa has not shown good progress in terms of reducing the prevalence of undernourishment compared to Asia and Latin
America. Ethiopia have also a larger share of the global burden of undernutrition. ${ }^{4}$ Under nutrition and food insecurity, secondarily to poor or untimely rainfall, are significant concerns in Ethiopia. Ethiopia has one of the highest number of undernourishment people among the countries in eastern African countries, with an estimated 31.4 million people undernourished in 2014. ${ }^{2}$ However, the country has demonstrated a significant decline in the prevalence of undernourishment over the period from 1990-92 to 2014-16. For example, the proportion of undernourished people in Ethiopia had declined by $74.8 \%$ from $74.8 \%$ in $1990-92$ to $32 \%$ in $2014-16 .{ }^{2}$ The most common macronutrient deficiencies in Ethiopia include stunting, wasting and underweight among children under five age, as well as chronic energy deficiency among women of reproductive age group. The rates of stunting and underweight have decreased over the past 15 year ${ }^{5,6}$ but remain high, with $40 \%$ of children under age five stunted $25 \%$ underweight. The progress in the reduction of stunting and underweight prevalence is not uniform across the regions, and pocket studies indicated a significant variability in the magnitudes within the country. Micronutrient deficiencies among children under five are also among the nutritional problems of public health importance. Vitamin A, iron, and iodine deficiencies greatly contribute to the burden of chiildhood morbidity and mortality. In addition to this, $52-73 \%$ of children of age group from six to 23 months are anaemic rate (EDHS, 2011). Breast feeding and complimentary feeding practices are essential to help the nutritional needs of children in the first years 
of life. In 2008, the World Health Organization(WHO) put a core set of eight indicator (i.e three breast feeding and five complimentary feeding) to help measure the appropriateness of feeding practices among children from six to 23 months. ${ }^{7}$ The eight core indicator include the initiation of breast feeding, exclusive breast feeding and continuing breast feeding for atleast one year and complimentary feeding which includes minimum dietary diversity, minimum meal frequency, minimum meal acceptable diet and consumption of iron rich foods. The objective of this study is to identify the effects of some predictor variables on nutritional status of child.

\section{Data and statistical model}

\section{Source of data}

This study is based on the 2011 Ethiopian Demographic and Health Survey (EDHS) data obtained from the Central Statistics Agency of Ethiopia. In this study, to assess the nutritional status of children, we have three anthropometric indices: weight-for-age, height-for-age and weight-for-height which are considered as the indicators of nutritional status.

\section{Variables of the study}

In this study socio-economic, demographic, health and environmental characteristics are to be proximate determinants of nutritional status. In our application on childhood nutritional status the variables are Weight-for-age (underweight) which is measured by $\mathrm{Z}$-score and body mass index(BMI). Body mass index is a measure of weight adjusted for height. It is calculated as weight in kilograms divided by the square of height in meters. ${ }^{8}$ These variables are continuous. Since the nutritional status of children(weight-for age) and body mass index are affected by a number of factors, the predictor variables to be analyzed in this study as determinants of nutritional status of children are grouped as socio-economic, demographic, health and environmental factors. These factors include the child's age, sex of child, birth order, preceding birth interval, duration of breast feeding, region, wealth index, mother education level, types of place of residence, number of household member, source of drinking water, ever had vaccination and children by choice not by chance.

\section{Methods}

Let $y$ be the random observation, $\Theta$ parameter space, and $\mathrm{p}$ densities the result will be

$$
p(\Theta \mid y)=\frac{p(y \mid \Theta) p(\Theta)}{p(y)}
$$

As $\Theta=\_1,2 \ldots . \mathrm{j}$ randomand $\mathrm{p}(\mathrm{y})=\int p(y \mid \Theta) p(\Theta) d \Theta$ is fixed and constant.

Thus the posterior distribution can be expressed as:

$$
p(\Theta \mid y) \propto p(y \mid \Theta) p(\Theta)
$$

Where $p(y \mid \Theta)$ is likelihood function, $p(\Theta \mid y)$ is posterior distribution, $p(\Theta)$ is prior distribution in this, we will analyze the effects of the different types of covariates on the response variables body mass index and nutritional status by using weight-for-age (underweight) Z-score as continuous variable with the assumption that each of the covariates has a linear effect on the response variable. Since we assume each covariate has a linear effect the approach we follow in this step is Bayesian Gaussian linear regression parametric approach.

\section{Bayesian generalized linear regression model}

When the effect of covariates on the response variables is linear we follow Bayesian Gaussian linear regression parametric approach. One of the most popular and useful tools in data analysis is the linear regression analysis model. Consider the normal linear regression model in which a response variable $y$ is related to one or more explanatory or predictor variables $X_{1}, \ldots, X_{p-1}$. For a random sample of $\mathrm{n}$ individuals, the model becomes:

$$
y_{\text {ïйі }} \beta_{\text {üüù }} \beta x+\beta x+\ldots+\beta_{-} x_{-}+\varepsilon
$$

where $p=19$ and $i=1, \ldots, 11,654$. We typically assume that $\varepsilon_{i}$ is normally distributed with mean 0 and variance $\sigma^{2}$, and $\varepsilon_{i}$ and $\varepsilon_{i}$ are independent of one another for $i \neq j$ i.e $\varepsilon_{i} \sim^{i i d} N\left(0, \sigma^{2}\right)$. In the Bayesian approach probability distributions are used to quantify uncertainty. Thus, in contrast to the frequentest approach, a joint probability model for response and parameters $\left(\beta, \sigma^{2}\right)$ is specified. The distribution of the dependent variable $y$ is specified conditional on the parameters ( $\beta$ and $\sigma^{2}$ )[8]. The analysts certainty or uncertainty about the parameter before the data analysis is represented by the prior distribution for the parameters $\left(\beta, \sigma^{2}\right)$. After observing the sample data $(y i, X i)$, the prior distribution is updated by the empirical data applying Bayes theorem, as in equation (1) and regression model in (2) we obtain equation (3).

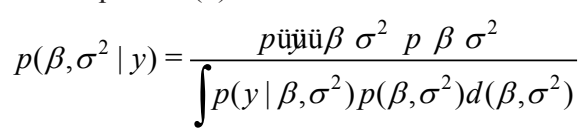

Yielding the so called posterior distribution $p\left(\beta, \sigma^{2} \mid y\right)$ of the parameters $\left(\beta, \sigma^{2}\right)$. For our specific application to multiple linear regression, we use a noncommittal, vague prior. For each regression coefficient, the prior distribution is a very broad normal distribution, with a mean of zero and a standard deviation that is large enough 1000.

\section{Prior distribution}

In Bayesian inference, the unknown function $f_{j}, \mathrm{j}=1, \ldots, \mathrm{p}$, the fixed effects parameters $\gamma=\left(\gamma_{1}, \ldots, \gamma_{r}\right)^{\prime}$ as well as the variance parameter $\tau^{2}$ are considered as random variables and have to be supplemented by appropriate prior assumptions. In the absence of any prior knowledge we assume independent diffuse priors $\gamma_{j} \propto$ const , $\mathrm{j}=1, \ldots, \mathrm{r}$ for the parameters of fixed effects. In this study, for the continuous covariates child age in month and Mother age at first birth those assumed to have a possibly nonlinear effect on the Z-score are modeled non-parametrically as cubic P-spline with second order random walk priors.

\section{The general form of the priors}

Suppose that $f=(f(1), \ldots, f(n))$ is the vector of corresponding function evaluations at observed values of $\mathrm{x}$. Then, the general form of the prior for $f$ is

$$
\ddot{u} i \ddot{u}^{2} \propto \quad\left(-\frac{1}{2 \tau^{2}} \quad, \quad\right)
$$

where, $K$ is a penalty matrix that penalizes too abrupt jumps between neighboring parameters. In most cases $f$ will be rank deficient; therefore the prior for $f$ would be improper. We choose an inverse gamma distribution with hyperparamters a and b, i.e.

$$
\tau^{2} \sim I G(a, b)
$$


with probability density function given by:

$$
p\left(\tau^{2} / a, b\right)=\left(\tau^{2}\right)^{-a-1} \exp \left(\frac{b}{\tau^{2}}\right)
$$

Common choices for $a$ and $b$ are $a=1$ and $b=0.005$ (or $b$ üüü $\quad$ ). Alternatively, one may take $a$ üü̉̈i

\section{Priors for fFixed effects}

As indicated above for the parameter vector $\gamma$ of fixed effects we choose a diffuse prior $\gamma_{j} \propto$ const $, \mathrm{j}=1, \ldots, \mathrm{r}$ Another choice would be to work with a multivariate Gaussian distribution $\gamma \sim N\left(\gamma_{0}, \sigma_{\gamma_{0}}\right)$. In this study, diffuse priors will be used for the fixed effects.

\section{Priors for continuous effects under linear setup}

Priors for the unknown functions $f_{1}, \ldots, f_{p}$ depend on the type of the covariates and on prior beliefs about the smoothness of $f_{j}$ . In the following we express the vector of function evaluations $f_{j}=\left(f_{j}\left(x_{1 j}\right), \ldots, f_{j}\left(x_{n j}\right)\right)$ ' of a function $f_{j}$ as the matrix product of a design matrix $X_{j}$ and a vector of unknown parameters $\beta_{j}$ s', i.e $f_{j}=X_{j}^{\prime} \beta_{j}$.

\section{First and second order random walk priors}

Let us consider the case of a continuous covariate $x$ with equally spaced observations ${ }_{m \leq}, \mathrm{i}=1, \ldots, \mathrm{m}, m \leq n$, then, $x_{(1)}<\ldots<x_{(m)}$ defines the ordered sequence of distinct covariate values. Here $m$ denotes the number of different observations for $x$ in the data set. A common approach in dynamic or state space models is to estimate one parameter $f(t)$ for each distinct $x(t)$; i.e., define, $f(t)=f\left(x_{(t)}\right)$ and let $f=(f(1), \ldots \ldots, f(m))^{\prime}$ denote the vector of function evaluation.

\section{Bayesian P-splines}

Any smoother depends heavily on the choice of smoothing parameter, and for $\mathrm{P}$ spline in a mixed (fixed and continuous) framework. A closely related approach for continuous covariates is based on the P-splines approach introduced by Eilers and Marx (1996). The basic assumption of this approach is that the unknown function $f_{j}$ can be approximated by a spline of degree 1 with equally spaced knots $x_{\text {min }}=\xi_{0}<\xi_{1}<\ldots<\xi_{r-1}<\xi_{r}=x_{\max }$ within the domain of $x_{j}$.

\section{Posterior inference}

In this case, the specific form of the posterior depends only on the parameterization of the regression terms in the model. Then, we use Markov Chain Monte Carlo (MCMC) simulations to draw samples from the posterior and statistical inference is done by means of Markov Chain Monte Carlo techniques in a full Bayesian setting. Now we restrict the presentation to models with predictor. Full Bayesian inference is based on the entire posterior distribution. Let $\alpha$ be the vector of all unknown parameters, then the posterior is given by.

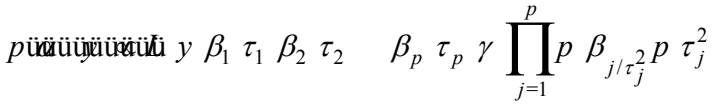

$$
\begin{aligned}
& \propto L\left(y, \aleph_{1} \aleph_{2},{ }_{2}, \ldots, \quad p, \quad p,\right)
\end{aligned}
$$

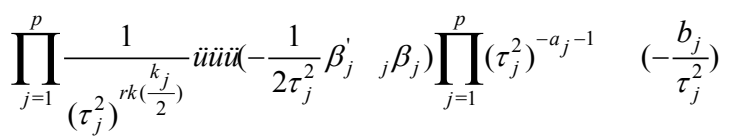

In a fully Bayesian approach, parameter estimates are generated by drawing random samples from the posterior via MCMC simulation techniques. The variance parameters $\tau_{j}^{2}$ can be stimated simultaneously with the regression coefficients $\gamma_{j}$ by assigning additional hyperprior to them. The most common assumption is, that the $\tau_{j}^{2}$ are independently inverse gamma distributed, i.e. $\tau_{j}^{2} \propto I G\left(a_{j}, b_{j}\right)$, with hyper-parameters $a_{j}$ and $b_{j}$ specified a prior. A standard choice is to use $a_{j}$ üü̈̈̈;

\section{Model diagnostic}

Once a model has been developed, we now would like to know how effective the model is in describing the outcome. This is referred to as goodness of fit. The most common ways of checking goodness of fit are: diagnosis for convergence and mixing and posterior-predictive check. For convergence tests, the researcher used time series plot.

\section{Model selection and comparison}

Model selection is to select the best model among several choices based on evaluation of the performance of the models. One immediate question is how to measure goodness of fit? There are many choices. The simplest one is residual sum of squares. ${ }^{9}$

$$
\sum\left(y_{i}-\hat{y}_{i}\right)
$$

Where $y_{i}$ is the observed responses and $\hat{y}_{i}$ is the fitted value. This measure is also called mean squared error, and commonly used as the default measure. For GLM, deviance is a quality of fit for a model that depends on the likelihood. The researcher skip the details and the underlying theory of the definition, but pointing out that the statistical software can compute the quantity automatically. The next question is why we should consider model selection? There are several reasons. First, people tend to believe or can understand simpler models with fewer predictors and less complicated structure. Second, one can certainly add more and more features into the model without screening and get better and better fit, till perfect fit, but the problem is over fitting. Remember that the researcher want to find the bestpredicting model not the best fitting model. In this study, AIC(Akaike Information Criterion), BIC(Bayesian Information Criterion), $\mathrm{CV}$ (Cross-Validation) and DIC (The deviance information criterion) are used to compare the linear frequent and the linear Bayesian approach with their smallest values to be better model. In this study, authors used R software(version 3.4.0) to analyze the determinant of factors under five years children nutritional status in Ethiopia.

\section{Results and discussion}

\section{Descriptive analysis of the data}

As one can see from Table 1, among the 11,654 cases examined in this study $32.8 \%$ of male children were underweight and $33.6 \%$ of female children were underweight. The highest prevalence of child malnutrition was observed among children whose preceding birth interval was less than 24 months (35\%) unlike to the lowest prevalence of child malnutrition which was recorded from children whose preceding birth interval is 48 and above $(31.9 \%)$. The proportion of underweight children as we can seen from Table 1, it is also differs by type of place of residence: urban and rural. Accordingly, higher numbers of underweight children $(33.6 \%))$ reside in rural areas, and relatively less numbers of underweight children $(31.2 \%$ ) reside in urban centers. The evidence given in Table 1 shows that the percentage nutritional statuses of children who had diarrhea recently 
seem to be high probability of underweight than those children which had no diarrhea recently. Likewise, one can observe that the percentages nutritional status of children who had fever recently seemed high probability of underweight than those children who have no fever recent $(33.9 \%, 33.1 \%$, respectively). The evidence given in Table 1 shows that the percentages nutritional status of children in households who had used other water sources seemed to be more underweight than those children in households which used water from public source. The family education level has also it's effect on the nutritional status of children that a mother with higher educational level(father education level) had a child with better nutritional status. Since the percentages of underweight is decreasing from no education to secondary and above education level. Children who had disease are affected by malnutrition e.g child who had cough is more affected than child who had not cough as we observe from Table 1.

Table I Summary of covariates with the response variable (weight-for-age)

\begin{tabular}{|c|c|c|c|c|}
\hline Covariates & Categories & Stunted & Wasted & Underweight \\
\hline \multirow[t]{11}{*}{ Region } & Tigray & $379(34.4 \%)$ & $35 \mathrm{I}(3 \mathrm{I} .8 \%)$ & $373(33.8 \%)$ \\
\hline & Affar & $358(36.5 \%)$ & $272(27.6 \%)$ & $355(36.0 \%)$ \\
\hline & Amhara & $420(35.6 \%)$ & $36 \mathrm{I}(30.6 \%)$ & $398(33.8 \%)$ \\
\hline & Oromia & $540(34.2 \%)$ & $492(31.2 \%)$ & $546(34.6 \%)$ \\
\hline & Somali & $377(42.3 \%)$ & $243(27.3 \%)$ & $27 \mid(30.4 \%)$ \\
\hline & Benishangul-Gumuz & $294(32.8 \%)$ & $304(34.0 \%)$ & $297(33.2 \%)$ \\
\hline & SNNP & $516(35.5 \%)$ & $466(32.0 \%)$ & $473(32.5 \%)$ \\
\hline & Gambela & $273(37.4 \%)$ & $228(31.3 \%)$ & $228(31.3 \%)$ \\
\hline & Harari & $24 I(40.3 \%)$ & $176(29.4 \%)$ & $181(30.3 \%)$ \\
\hline & Addis Ababa & $143(39.0 \%)$ & $103(28.1 \%)$ & $121(33.0 \%)$ \\
\hline & Dire Dawa & $226(35.8 \%)$ & $191(30.2 \%)$ & $215(34.0 \%)$ \\
\hline Place of residence & Urban & $697(39.8 \%)$ & $507(29.0 \%)$ & $547(31.2 \%)$ \\
\hline \multirow[t]{5}{*}{ Wealth index } & Poorest & $1172(36.4 \%)$ & $950(29.5 \%)$ & $1100(34.1 \%)$ \\
\hline & Poorer & $663(35.1 \%)$ & $607(32.1 \%)$ & $621(32.8 \%)$ \\
\hline & Middle & $565(33.4 \%)$ & $536(31.7 \%)$ & $589(34.9 \%)$ \\
\hline & Richer & $602(35.7 \%)$ & $537(31.8 \%)$ & $548(32.5 \%)$ \\
\hline & Richest & $765(39.8 \%)$ & $557(29.0 \%)$ & $600(31.2 \%)$ \\
\hline \multirow[t]{2}{*}{ Sex } & Male & $1918(36.2 \%)$ & $1646(31.0 \%)$ & $|74|(32.8 \%)$ \\
\hline & Female & $1849(36.3 \%)$ & $|54|(30.2 \%)$ & $1717(33.6 \%)$ \\
\hline \multirow[t]{2}{*}{ Children by choice } & No & $3027(36.1 \%)$ & $2559(30.5 \%)$ & $2798(33.4 \%)$ \\
\hline & Yes & $735(36.4 \%)$ & $628(31.1 \%)$ & $659(32.6 \%)$ \\
\hline \multirow[t]{3}{*}{ Mother edu.level } & No education & $2665(36.6 \%)$ & $2187(30.0 \%)$ & $2427(33.3 \%)$ \\
\hline & Primary & $900(34.5 \%)$ & $833(31.9 \%)$ & $875(33.1 \%)$ \\
\hline & Secondary and above & $202(38.5 \%)$ & $167(31.8 \%)$ & $156(29.7 \%)$ \\
\hline \multirow[t]{3}{*}{ Father edu.level } & No education & $1903(35.8 \%)$ & $1604(30.1 \%)$ & $1816(34.1 \%)$ \\
\hline & Primary & $1339(35.5 \%)$ & $1193(31.6 \%)$ & $1244(32.9 \%)$ \\
\hline & Secondary and above & $442(39.2 \%)$ & $338(30.0 \%)$ & $347(30.8 \%)$ \\
\hline \multirow[t]{2}{*}{ Drinking water } & Public & $1607(36.7 \%)$ & $1324(30.2 \%)$ & 1447(33.1\%) \\
\hline & Others & $2158(35.8 \%)$ & $1862(30.9 \%)$ & $2010(33.3 \%)$ \\
\hline \multirow[t]{3}{*}{ Ever had vaccination } & No & $881(38.9 \%)$ & $675(29.8 \%)$ & $708(31.3 \%)$ \\
\hline & Yes & $1990(35.6 \%)$ & $1693(30.3 \%)$ & $1912(34.2 \%)$ \\
\hline & Rural & $3070(35.4 \%)$ & $2680(30.9 \%)$ & $2911(33.6 \%)$ \\
\hline Had diarrhea recently & No & $322 I(36.6 \%)$ & $2709(30.8 \%)$ & $2867(32.6 \%)$ \\
\hline
\end{tabular}




\begin{tabular}{lllll} 
Table Continued & \multicolumn{1}{l}{} \\
\hline Covariates & Categories & Stunted & Wasted & Underweight \\
\hline \multirow{3}{*}{ Had child fever } & Yes & $530(33.3 \%)$ & $475(29.8 \%)$ & $588(36.9 \%)$ \\
& No & $3028(36.3 \%)$ & $2548(30.6 \%)$ & $2757(33.1 \%)$ \\
Had cough recently & Yes & $719(35.3 \%)$ & $627(30.8 \%)$ & $691(33.9 \%)$ \\
& No & $3029(36.5 \%)$ & $2507(30.2 \%)$ & $2757(33.2 \%)$ \\
Preceding birth interval & Yes & $723(34.6 \%)$ & $674(32.2 \%)$ & $695(33.9 \%)$ \\
& less than 24 & $1022(36.6 \%)$ & $825(29.5 \%)$ & $969(35.0 \%)$ \\
& $24-48$ & $999(37.0 \%)$ & $839(31.1 \%)$ & $945(33.8 \%)$ \\
Breast feeding & Above 48 & $946(34.2 \%)$ & $853(30.8 \%)$ & $861(31.9 \%)$ \\
& Never breastfed & $61(39.1 \%)$ & $45(28.8 \%)$ & $50(35.1 \%)$ \\
& Ever breastfed(not cur) & $1983(36.0 \%)$ & $1689(30.7 \%)$ & $1832(32.3 \%)$ \\
& Still breast feeding & $1700(36.1 \%)$ & $1442(30.6 \%)$ & $1570(30.3 \%)$ \\
\hline
\end{tabular}

From Table 2 the highest malnourished region are SNNP, Oromia, Gambela, Affar and Tigray. From all of these region the smallest malnourished region is Harari. The place of residence has also its impact as result the highest malnourished lives in rural comparing with urban.

Table 2 Summary of Covariates with the response variable(Body mass index)

\begin{tabular}{|c|c|c|c|}
\hline Covariates & Categories & malnourished & well-nourished \\
\hline \multirow[t]{11}{*}{ Region } & Tigray & $550(49.7 \%)$ & $556(50.3 \%)$ \\
\hline & Affar & $494(49.8 \%)$ & $498(50.2 \%)$ \\
\hline & Amhara & $558(47.3 \%)$ & $622(52.7 \%)$ \\
\hline & Oromia & $795(50.1 \%)$ & $793(49.9 \%)$ \\
\hline & Somali & $423(47.3 \%)$ & $47 \mid(52.7 \%)$ \\
\hline & Benishangul-Gumuz & $472(52.6 \%)$ & $426(47.4 \%)$ \\
\hline & SNNP & $782(53.5 \%)$ & $680(46.5 \%)$ \\
\hline & Gambela & $329(44.9 \%)$ & $403(55.1 \%)$ \\
\hline & Harari & $249(41.6 \%)$ & $349(58.4 \%)$ \\
\hline & Addis Ababa & $157(42.7 \%)$ & $211(57.3 \%)$ \\
\hline & Dire Dawa & $311(49.0 \%)$ & $324(51.0 \%)$ \\
\hline \multirow[t]{2}{*}{ Place of residence } & Urban & $802(45.6 \%)$ & $955(54.4 \%)$ \\
\hline & Rural & $4318(49.7 \%)$ & $4378(50.3 \%)$ \\
\hline \multirow[t]{5}{*}{ Wealth index } & Poorest & $1610(49.8 \%)$ & $|62|(50.2 \%)$ \\
\hline & Poorer & $925(48.5 \%)$ & $982(51.5 \%)$ \\
\hline & Middle & $867(51.1 \%)$ & $830(48.9 \%)$ \\
\hline & Richer & $834(49.4 \%)$ & $855(50.6 \%)$ \\
\hline & Richest & $884(45.8 \%)$ & $1045(54.2 \%)$ \\
\hline \multirow[t]{2}{*}{ Sex } & Male & $2654(49.8 \%)$ & $2664(50.2 \%)$ \\
\hline & Female & $2463(48.0 \%)$ & $2673(52 \%)$ \\
\hline \multirow[t]{2}{*}{ Source of drinking water } & public & $214 \mathrm{I}(48.7 \%)$ & $2252(51.3 \%)$ \\
\hline & others & $2975(49.1 \%)$ & $308 I(50.9 \%)$ \\
\hline Had diarrhea & No & $4290(48.6 \%)$ & $4543(51.4 \%)$ \\
\hline
\end{tabular}




\begin{tabular}{llll} 
Table Continued & & \\
\hline Covariates & Categories & malnourished & well-nourished \\
\hline \multirow{3}{*}{ Had child fever } & Yes & $51.5(823 \%)$ & $775(48.5 \%)$ \\
Ho cough recently & Yes & $4064(48.6 \%)$ & $26644301(51.4 \%)$ \\
No & Yes & $1037(50.7 \%)$ & $1009(49.3 \%)$ \\
preceding birth interv & less than 24 & $4037(48.5 \%)$ & $4285(51.5 \%)$ \\
& $24-48$ & $1076(51.1 \%)$ & $1028(48.9 \%)$ \\
& above 48 & $1407(50.1 \%)$ & $1400(49.9 \%)$ \\
Duration of bf & never breastfed & $1319(48.8 \%)$ & $1386(51.2 \%)$ \\
& ever bf, not currently & $1394(46.1 \%)$ & $8613(53.9 \%)$ \\
& still bf & $2634(47.6 \%)$ & $2897(52.4 \%)$ \\
\hline
\end{tabular}

\section{Bayesian generalized linear regression model}

Results of generalized linear regression analysis weight-for-age revealed that, the intercept is estimated to be -692.832 . It can be interpreted as the value you would predict for weight for age if all predictor variables are set to minimum. We would expect an average Z-sore value of approximately -692.832 for weight for age (under weight). Results of generalized linear model analysis weight for age shows that sex of child is significant(estim -145.487, p-value 0.0315 ) which indicate that, Weight-for-age(underweight) of a female is lowered by 145.487 as compared to a male child. Children by choice not by chance is significant( estim -214.050, p-value 0.0432) that is Underweight of children by choice not by chance is lowered by 214.0.050 as compared to children by chance. Mother education level at secondary and above is significant $10 \%$ level. And it is positively related to weight-for-age of children (estim 838.0805, p-value 0.0873). Weight-for-age declines with increasing mother education level. Ever had vaccination is significant(estim -194.487, p-value 0.0270). Weight-for-age of children who had ever vaccination is lowered by 194.487 as compared to no had never vaccination. Had cough is significant. Weight-for-age of children who had cough is increased by 282.807 as compared to who had no cough. Duration of breast feeding who had ever breasted, not currently breastfeeding is positively related to weight-for-age (etim 314.196, $\mathrm{p}$-value $=0.0655$ ), significant at $10 \%$ level. Weight-for-age declines with increase in duration of breast feeding of a child. Results of generalized linear model for frequentist approach for body mass index shows that the intercept value is approximately $-553.208 \beta_{0}$, the y-intercept, can be interpreted as the value you would predict for body mass index if all predictor variables are zero. We would expect an average value of approximately -553.208 for body mass index (malnourished). However, this is only a meaningful interpretation if it is reasonable that all the predictors equal to zero, and if the data set actually included values for all predictors that were near 0 . If neither of these conditions are true, then $\beta_{0}$ really has no meaningful interpretation. It just shift the regression line in the right place. The current study revealed that, Child age in month is significant at $10 \%$ level. Body mass index of child is negatively related to child age(estm 6.340, p-value 0.0812). Which implies as child age increases the body mass index is deceased. Succeeding birth interval is positively related to body mass index (etim 6.558, p-value $=0.0722$ ), significant at $10 \%$ level. Body mass index increase with increasing in succeeding birth interval of a child. Sex of child (gender) is significant. Body mass index of a female is lowered by 140. as compared to a male child. Children by choice not chance is significant. Body mass index of child is negatively related to children by choice not chance. Child ever had vaccination is significant. Body mass index of child is negatively related to ever had vaccination. Child who had cough is significant at $10 \%$. Body mass index of child is positively related to child who had cough. Duration of beast feeding is significant. Body mass index of child is positively to duration of breast feeding.

Table 3 gives results for the fixed effects (categorical covariates) on the nutritional status of children under age five in Ethiopia. The output gives posterior means along with their standard deviations and $95 \%$ credible intervals. Since the $95 \%$ confidence interval do not include zero, sex of child, child by choice not by chance, ever had vaccination and had cough were found statistically significant at $5 \%$ significance level. But, regions, mother education level, source of drinking water and duration of breast feeding were found statistically insignificant. One can observe that child who had vaccinated contributes to better nourishment for children under five age. In this study, the relative chance to be underweight for the children was found to decrease with the increase of vaccination for children. The children who had no vaccination were more likely to be malnourished as compared to children ever had vaccination. And also the analysis shows that female children are better nourished than male children. On the other hand, one can observe that underweight is higher for children who had cough than child who had no cough. So children who had cough should be treated at early stage. We can see the output matches up with frequentest(traditional) approach. As the sample size increase the result should converge to the same values. One of the benefits of the Bayesian perspective (for any analysis) is that it allows us to make credible interval statements. Credible intervals are similar to confidence intervals, but in the Bayesian framework, the interval REALLY IS believed to contain the true population parameter. For instance: a $95 \%$ credible interval for a parameter being estimated is interpreted as, there is a $95 \%$ probability that the actual parameter is included in that interval. This is because the interval is based on information from the posterior distribution, of for instance, one of the predictor's coefficient posterior distribution. For example, we can say there is a $95 \%$ probability that the true population value of the Child's sex (Female) coefficient is between -271.255 and -11.280 . 
Table 3 Summary of Bayesian generalized linear model fixed effects estimation results of weight-for-age

\begin{tabular}{lllllc}
\hline Intercept) & Mean & Sd & $\mathbf{2 . 5 0 \%}$ & $\mathbf{5 0 \%}$ & $\mathbf{9 7 . 5 0 \%}$ \\
\hline & -37.017 & 172.848 & $-380.80 I$ & $-35.5 I 3$ & 296.638 \\
as.factor (Region I) Tigray & -235.028 & 136.785 & -498.682 & -236.257 & 35.935 \\
as.factor (Schild) Female & -140.934 & 66.113 & -271.255 & -141.446 & -11.28 \\
as.factor (Childbychoice) Yes & -238.769 & 100.714 & -443.776 & -236.86 & -47.297 \\
as.factor (MEDL) Secondary and above & 829.133 & 443.42 & -26.276 & 832.555 & 1689.62 \\
as.factor (SDW) Others & 103.306 & 67.58 & -26.675 & 103.055 & 236.557 \\
as.factor (EV) Yes & -180.055 & 80.879 & -336.825 & -181.197 & -21.63 \\
as.factor (HC)Yes & 217.882 & 93.165 & 38.254 & 219.401 & 397.688 \\
as.factor (DBFa) Ever breastfed, not current & 276.466 & 164.331 & -39.01 & 275.631 & 598.303 \\
\hline
\end{tabular}

Scale estimate: 2178410

$\mathrm{N}=1969 \mathrm{DIC}=1986.847 \mathrm{pd}=8.922508 \mathrm{AIC}=28753.8$

method $=$ STEP family=gaussian iterations $=10000$ step $=2$

From Table 4 we also observe that number of significant variables are the same for weight for age and as the result those variables are affect body mass index. Body mass index of child sex(female) is lowered by -138.5870 comparing to male child which indicate the decrement of body mass index is highly observed in female children that can make female more susceptible to illness, impair the growth and development, cause infertility and the raise for different disease including death. Likewise from Table 4 child who had cough faces high body mass index comparing to child who had not cough and child ever vaccinated faces low body mass index. Which indicate only vaccination is not enough as result children should get sufficient macro nutrients. Generally, the Bayesian generalized linear model satisfy the normal assumption of the residual. the models in Bayesian approach MCMC post estimation diagnosis, it is also possible to extract sampling paths of parameters with function samples(), or to plot the samples directly. For instance, coefficient sampling paths for term $\mathrm{sx}(\mathrm{SBI})$ are displayed which is important to check the model convergence in the time series plot.

Table 4 Summary of Bayesian generalized linear model fixed effects estimation results: for BMI

\begin{tabular}{|c|c|c|c|c|c|}
\hline (Intercept) & Mean & Sd & $2.50 \%$ & $50 \%$ & $97.50 \%$ \\
\hline & 167.046 & 222.901 & -262.467 & 168.528 & 601.062 \\
\hline Child age & -6.1193 & 3.5448 & -13.1032 & -6.1044 & 0.7738 \\
\hline SBI & 7.0981 & 3.5063 & 0.3645 & 7.0083 & 14.1063 \\
\hline as.factor (Region I) Tigray & -199.706 & 135.52 & -475.312 & -199.459 & 69.1336 \\
\hline as.factor (RegionI) SNNP & $|4| .205$ & 89.4776 & -34.7017 & 143.12 & 317.365 \\
\hline as.factor (Schild) Female & -138.587 & 65.7587 & -268.593 & -137.592 & -11.8307 \\
\hline as.factor (Childbychoice) Yes & -220.97 & 99.9777 & $-4 \mid 3.363$ & -221.502 & -24.937 \\
\hline as.factor (MEDL ) Secondary and above & 772.072 & 441.918 & -111.42 & 781.612 & 1616.64 \\
\hline as.factor (EV) Yes & -163.949 & 80.9812 & -327.144 & -163.562 & -2.8443 \\
\hline as.factor $(\mathrm{HC})$ Yes & 207.696 & 92.5048 & 25.2276 & 207.318 & 389 \\
\hline as.factor (DBFa) Ever breastfed, not current & 268.439 & 163.619 & -50.7223 & 266.995 & 591.912 \\
\hline
\end{tabular}

Scale estimate: 2126770

$\mathrm{N}=1969 \mathrm{DIC}=|990.78 \mathrm{I} \mathrm{pd}=| 0.88936 \mathrm{AIC}=287 \mid 0.6$

method $=$ STEP family=gaussian iterations $=10000 \mathrm{step}=2$ 


\section{Model comparison}

We analyzed the DHS data set using two different approaches. It is reasonable to ask which type of model is better. The two models are listed in the following:

\section{Model $1=$ Generalized linear regression model in frequentest}

Model 2 = Generalized linear regression model in Bayesian Model selection is an important part of statistical analysis, and indeed is a central to the pursuit of science in general. Many authors have examined this question, rom both frequentist and Bayesian perspective, and many tools o selecting the "better model". The models proposed in this study are quite general and the model building process can be quite challenging. Currently, an automated procedure for Bayesian model selection is not available. However, a few recommendations are possible. Users should try to incorporate everything that is theoretically possible and different Bayesian models could be compared via the DIC. ${ }^{10}$

From Table 5, when we compare Model 1 and Model 2 via AIC, the most interesting fact here is that the better models in terms of AIC. Model 2 (Generalized linear regression model in Bayesian approach) since they have less value (28753.8) and (28710.6) and they are chosen by the data analyst. The point is that models 2 are a better models than models 1 (Gaussian linear regression model in frequentest approach).

Table 5 Summary result of models comparison criteria for weight-for-age on the left and body mass index on right hand side

\begin{tabular}{llllllll}
\hline & AIC & GCV & DIC & & AIC & GCV & DIC \\
\hline Model I & 34378 & - & - & Model I & 34334 & - & - \\
Model 2 & 28753.8 & - & 1986.847 & Model 2 & 28710.6 & - & 1990.781 \\
\hline
\end{tabular}

\section{Discussion}

This study was intended to identify the determinants of the nutritional status of children under five years old in Ethiopia based on EDHS 2011 data. The nutritional status was measured by the weight-for-age and body mass index is measured by weight/height. Accordingly, Bayesian Generalized linear model analysis on underweight was employed to identify flexibly the effect of covariates on nutritional status of the children. In this study, underweight was analyzed based on the modified anthropometric measurement indicators of the nutritional status of children calculated using new growth standards published by the World Health Organization in 2006. The results obtained are discussed as follows. The total number of children covered in the present study was 11,654, among which $34.4 \%$ were underweight. The Bayesian Generalized linear model analysis revealed that the covariates: sex of child, children by choice not by chance, ever had cough and ever had vaccination were found statistically significant. But, region, mother education level, source of drinking water and duration of beast feeding were found statistically insignificant. In this section, sex of child (female) was also played statistically significant role in identify children malnutrition in Ethiopia. This finding is consistent with the study in the context of child's malnutrition study in Ethiopia, ${ }^{11}$ which reported that child's sex has a strong and significant effect on nutritional status of children. Under body mass index for Bayesian Generalized linear model analysis covariates like succeeding birth interval, sex of child, children by choice not by chance, ever had vaccination and cough were found statistical significant. But, child age in month, region, mothers education level and duration of breast feeding were statistically insignificant which is some what consistent with. ${ }^{12}$ The findings of this study also showed that children who had cough are vulnerable to malnutrition problem than those who had not. This finding is consistent with other studies. ${ }^{13,14}$ This may be due to the fact that cough accelerates the onset of malnutrition by reducing food intake. In consequence, these results increased risk of childhood diseases, which contribute to malnutrition. As shown in the analysis, urban children were less likely to be malnourished than their rural counterparts because the quality of health environment and sanitation is better in urban areas, whereas, the living condition in rural areas were associated with poor health condition, and lack of personal hygiene, which were the risk factors in determining malnutrition. This is contradict with some studies, where place of residence has a statistical insignificant effect on children nutritional status. ${ }^{15,16}$ The findings of this study also showed that children who had ever vaccinated are not such vulnerable to malnutrition problem than those who had not vaccinated. This result is unique from all of the previous research since ever had vaccination is insignificant for the previous one. ${ }^{15-39}$

\section{Conclusion}

The results found that children living in the Affar and Oromia region of Ethiopia are associated with weight for age (underweight). While the children which are living in Harari and Somali are less associated with underweight comparing with other regions. Determinants that affects levels of nutritional status of children have been explored using different models like Generalized linear models in frequentest and Bayesian approaches. In these model, the analysis shows that child sex, children by choice not by chance, vaccination, source of drinking water, duration of breast feeding and cough important fixed factors which affect the weight for age of children in Ethiopia. Variables like succeeding birth interval, child sex, children by choice not by chance, vaccination, and cough are also affect body mass index. Mother education level, which is related to wealth index, is a determinant of good child care knowledge and practices. In this study, the education attainment of mothers does not have a significant effect on the underweight of a child in Ethiopia in separate analysis. This result is against the suggestion that an educated mother assumes the responsibility of taking a sick child to receive care health. Mother education level, which is related to succeeding birth interval have also determine the body mass index since they are significant effect.

\section{Recommendations}

In addition to the efforts being made to reduce malnutrition in general, specific attention should be given to reduce the prevalence of malnutrition by taking the following in to consideration. The concerned body: 
a. Should give due attention to the high risk factors identified as contributing high risk of malnutrition in children under five years of age due emphasis.

b. Should be given better source of water for community in more facilitated manner.

c. And also male and female children should be equally treated and have similar care and support since female children were more likely to be underweight than male children in Ethiopia.

d. Finally, further findings should be incorporating for additional risk factors which are associated with childhood malnutrition in Ethiopia regional wise.

\section{Acknowledgments}

The authors gratefully acknowledge Central Statistics Agency for providing the data. This work was financially supported by the College of Natural Science, Hawassa University.

\section{Conflicts of interest}

Author declares that there is no conflicts of interest.

\section{References}

1. Stevens Ga, Finucane MM, Paciorek CJ, et al. Trends in mild, moderate, severe stunting and underweight, and progress towards MDG 1 in 141 developing countries: A systematic analysis of population representative data. The Lancet. 2012;380:824-834.

2. The state of Food Insecurity in the world. Rome, Italy: FAO; 2015.

3. Blach RE, Vectoria CG, Walker SP, et al. Maternal and child under nutrition and overweight in low-income and middle-income countries. The Lancet. 2013;382(9890):427-451.

4. The State of Food Insecurity in the World 2012. Economic growth is necessary but not sufficient to accelerate reduction of hunger and malnutrition. Rome: FAO; 2012.

5. Central Statistics Agency Ethiopia. Ethiopia Mini demographic and Health Survey. Ethiopia: Addis Ababa; 2014.111 p.

6. Central Statistics Agency Ethiopia. Ethiopian Demographic and Health Survey. USA: Central Statistics Agency and ORC Macro; 2011. 452 p.

7. World Health Organization. The world health report 2006 - working together for health. USA: WHO; 2008.

8. Sopher A, Shen W, Pietrobelli A. Pediatric body composition methods. In: Heymsfield, Lohman, Wang, Going, editors. Human Body Composition. Champaign, IL: Human Kinetics; 2005. pp. 129-140.

9. Loader C. Local Regression and Likelihood. New York: Springer; 1999

10. Spiegelhalter D, Best N, Carlin B, et al. Bayesian Measures of Models Complexity. Journal of the Royal Stat. 2002;(64):1-34.

11. Dereje D. Statistical Analysis of Determinants of Nutritional Status of Children under Age Five: A Case Study of Hawassa Zuria Wereda in Sidama Zone, SNNPR, Ethiopia. 2011.

12. Mohammad A. Gender Differentials in Mortality and Under nutrition in Pakistan: Peshawar. 2008.

13. Khaled K. Child Malnutrition in Egypt Using Geo-additive Gaussian and Latent Variable Models. Am J Trop Med Hyg. 2010;82(4):653-663.

14. Birhan Fetene. Determinants of Nutrition and Health Status of Children in Ethiopia: A Multivariate Multilevel Linear Regression Analysis. Thesis: Addis Ababa University; 2010. 94 p.
15. Kandala NB, S Lang, S Klasen, et al. Semiparametric Analysis of the Socio-Demographic Determinants of Undernutrition in Two African Countries. Research in Official Statistics, EUROSTAT. 2006;4(1):81-100.

16. Woldemariam Girma, Timotiows Genbo. Determinants of the Nutritional Status of Mothers, and Children in Ethiopia. USA: ORC Macro. 2002.

17. Burnham KP, Anderson DR. Model Selection and Multi-model Inference, A Practical Information Theoretic Approach. New York: Springer; 2002. $266 \mathrm{p}$.

18. Charles H, Dorosty R, Reilly J. Coexistence of social inequalities in under nutrition and obesity in preschool children: population based cross sectional study. UK: Caledonian University; 2008. 5 p.

19. Christiaensen L, Alderman H. Malnutrition in Ethiopia: Can Maternal Knowledge Augment the role of Income? World Bank. 2001.287 p.

20. Congdon P. Bayesian Statistical Modelling. England: Wiley; 2001.596 p

21. Dejen A. The determinants of nutritional status of children in Ethiopia using Multivariate analysis. AAU: MSc thesis in applied statistic; 2008. $96 \mathrm{p}$.

22. FMOH. Federal Democratic Republic of Ethiopia Ministry of Health Health Sector Transformation Plan. 2016

23. Haile M. Weather patterns, food security and humanitarian response in sub-saharan Africa. Philos Trans R.Soc Land B Biol Sci. 2005;360:21692182.

24. Kloss H, Lindtjorn B. Malnutrition during Recent Famines in Ethiopia Northeast African studies. 1994;1:121-136.

25. Micheal Adam. The relationship between nutritional status of children and KAP of mothers' feeding their children in a Rural Community: Hawa-Michael, Bugna Woreda. 2000.

26. Muller O, Krawinkel M. Malnutrition and Health in Developing Countries. CMAJ. 2005;173(3):279-286.

27. Raach AW. A Bayesian Semiparametric Latent Variable Model for Binary, Ordinal and Continuous response, Dissertation, Department of Statistics. Germany: University of Munich; 2005.

28. RE Black, CG Victora, SP Walker, et al. Maternal and Child Undernutrition and Overweight in Low-Income and Middle-Income Countries. The Lancet. 2013;382(9890):427451.

29. Rahman A, Chowdhury S. Determinants of Chronic Malnutrition Among Preschool Children in Bangladesh. Journal of Biosocial Science. 2007;39(2):161-173.

30. Sasha F. An Analysis of Under-Five Nutritional Status in Lesotho: The Role of Parity Order and Other Socio-Demographic Characteristics. South Africa: University of The Witwatersrand, Johannesburg; 2009.

31. Sereebutra P, Solomons N, Aliyu MH, et al. Socio-Demographic and Environmental Predictors of Childhood Stunting in Rural Guatemala. Nutrition Research. 2006.

32. Silva P. Environmental factors and children malnutrition in Ethiopia Policy Research Working Paper Series from The World Bank No 3489.

33. Taye A, Maiam DH, Murray V. Interim report: Review of evidence of the health impact of famine in Ethiopia. Perspect Public Health. 2010;130(5):222-226.

34. Pelletier DL, EA Frongillo, DG Schroeder, et al. A methodology for estimating the contribution of malnutrition to child mortality in developing countries. Journal of Nutrition.1994;124(124):2106S-2122S.

35. World Health Organization. Comparative risk assessment of nutritional status of children. USA: WHO; 2002 
36. World Health Organization. The world health report 2006 - working together for health. USA: WHO; 2006.

37. World Bank. Levels and trends in child malnutrition. New York: UNICEF; 2019. pp. 5-36.

38. Yimer G. Malnutrition among children in southern Ethiopia: Levels and risk factors. Ethiopian Journal of Health Development. 2000;14(3):283292.
39. Zewditu G, Kelbessa G, Timotiows, et al. Review the status of malnutrition and trend in Ethiopia. Ethiopian Journal of Health and Development. 2001;15(2):55-74. 